\title{
Producción de tableros de partículas con Bolaina (Guazuma crinita Mart) procedente de una plantación de cuatro años
}

\author{
Milciades Leonidas Miguel Castro ${ }^{1}$, Setsuo Iwakiri ${ }^{2 \star}$, Rosilani Trianoski ${ }^{2}$, Héctor Enrique Gonzales \\ Mora $^{1}$, Cynthia Rosario Miguel Córdova ${ }^{1}$

\begin{abstract}
${ }^{1}$ Universidad Nacional Agraria La Molina Departamento de Industrias Forestales. Lima, Perú.
${ }^{2}$ Departamento de Engenharia e Tecnologia Florestal, Universidade Federal do Paraná, Curitiba, Paraná, Brasil.
\end{abstract}

\begin{abstract}
RESUMEN Se elaboraron tableros de partículas de tres capas, $12 \mathrm{~mm}$ de espesor y 0,65 g.cm ${ }^{-3}$ de densidad con madera de "bolaina" (Guazuma crinita Mart), de una plantación de cuatro años localizada en Neshuya, - Ucayali, Perú. Las partículas fueron encoladas con Urea Formaldehído. Se evaluaron las propiedades físicas y mecánicas de los tableros según la metodología propuesta por las Normas Europeas (EN). Los valores de masa específica y pH de la madera son los adecuados para la elaboración de tableros de partículas; el hinchamiento sin parafina a las 2 y 24 horas de inmersión en agua fue 26,45\% y 36,40\% respectivamente; con parafina a las 2 horas el hinchamiento disminuyo en un 5.29\% sin embargo a las 24 horas de inmersión en agua el hinchamiento se incrementó en $1.88 \%$. La flexión estática promedio sin parafina fue de 15,55 MPa, valor cercano al encontrado con parafina. La tracción perpendicular promedio sin parafina fue de $0,97 \mathrm{MPa}$ con un descenso de $15.46 \%$ cuando de utilizó parafina. La evaluación de los resultados obtenidos, permiten apreciar que es posible fabricar tableros de partículas con la madera bolaina de cuatro años de edad.
\end{abstract}

Palabras claves: partículas de madera; tableros de partículas; urea formaldehido; parafina.

\section{Production of particleboards with Bolaina (Guazuma crinita Mart) from a four-year-old plantation}

\begin{abstract}
Production of particle boards with Bolaina (Guazuma crinita Mart) from a four-year-old plantation. Three-ply particle boards, $12 \mathrm{~mm}$ thick and $0,65 \mathrm{~g} . \mathrm{cm}^{-3}$ of density, were made using wood (Guazuma crinita Mart), "bolaina", from a fouryear plantation located in Neshuya - Ucayali. The particles were glued with a formaldehyde urea. The physical and mechanical properties of the boards were evaluated according to the methodology proposed by the European Standards for each test. The values of the specific mass and $\mathrm{pH}$ of the wood are suitable for the manufacture of particle boards, swelling without paraffin at 2 and 24 hours of immersion in water was $26,45 \%$ and $36,40 \%$ respectively with paraffin at 2 hours the swelling decreased by $5.29 \%$ however at 24 hours of immersion in water the swelling increased by $1.88 \%$. The mean static bending without paraffin was 15.55 $\mathrm{MPa}$, a value close to that found with paraffin. The internal bond without paraffin was $0.97 \mathrm{MPa}$ with a decrease of $15.46 \%$ when using paraffin. The evaluation of the results obtained shows that it is possible to manufacture particle boards use with 4-yearold bolaina.
\end{abstract}

Keywords: wood particle; particleboard; urea-formaldehyde; paraffin.

\section{Introducción}

Según Flores (2018), la Guazuma crinita Mart. (Bolaina) usualmente crece en agrupaciones casi homogéneas llamadas "bolainales", pero también crece asociado con otras especies pioneras como Schizolobium amazonicum, Croton matourensis, Cecropia sp., Calycophyllum spruceanum, Cordia alliodora, Inga spp., entre otras especies. Menciona además que es deseable para plantaciones forestales por su rápido crecimiento con un período de cosecha de aproximadamente 7 años, su copa rala, su capacidad de 
manejo de rebrotes hasta 3 oportunidades, el color claro de su madera, la ausencia de resinas en la madera y su notable capacidad de regeneración.

El Instituto de Investigación de la Amazonía Peruana (IIAP), citado por Ramos-Huapaya et al. (2016), añaden que es una especie amazónica de alta abundancia natural, baja tolerancia a la competencia y alta capacidad de rebrote. Putzel et al. (2013) resaltan su rápido crecimiento, alcanzando un incremento en su diámetro promedio de $4,8 \mathrm{~cm} / \mathrm{año}$, lo que permite utilizarla en postes luego de 2 años y como madera aserrada después de 11 a 12 años. Wigthtman et al. (2006), manifiesta que se han realizado plantaciones exitosas a pequeña escala en Selva Alta y Selva Baja (Ucayali - Perú). En Ucayali las mejores plantaciones han estado en zonas aluviales o de alta precipitación y en suelos que van de franco-arcillosos a arcillosos. En estos sitios, los árboles han alcanzado alturas de 10 metros a los 4 años después de plantados.

Respecto a su importancia económica como materia prima para productos maderables, SERFOR (2018) y SERFOR (2019), registran una producción de madera rolliza de Bolaina para el año 2016 de $36465 \mathrm{~m}^{3}$, que representa el 2,52\% de la producción nacional de madera rolliza, y para el año 2017 su producción se incrementó a 3,42\%.

En relación a las propiedades de la madera como materia prima para la fabricación de tableros aglomerados, Marra citado por Trianoski et al. (2015), mencionan que las propiedades anatómicas, físicas y químicas de la madera pueden influenciar en la fabricación de productos encolados.

Maloney (1993), menciona que el tipo de madera tiene una influencia en la masa específica, la humedad del tablero y la formulación del adhesivo. Las características de la madera que influyen en la calidad del tablero, según Moslemi (1974) e Iwakiri (2010), son la masa específica de la madera, $\mathrm{pH}$, humedad y contenido de extractivos. Asimismo et al. citado por Moslemi (1974), señalan que conforme se incrementa la masa específica de la madera, para una misma masa específica del tablero, el módulo de rotura (MOR), disminuye. Esto se explica porque una partícula de baja masa específica tendrá mayor relación de compactación y genera una mejor unión adhesiva por una mayor eficiencia y exposición de cola. Maloney (1993) señala que se prefieren especies cuyo rango de masa especifica básica varíe entre 0,3 a $0,5 \mathrm{~g} . \mathrm{cm}^{-3}$, por su mejor comportamiento a la compactación.

Respecto al pH y extractivos de la madera, Marra citado por Trianoski et al. (2015) y afirma que estos parámetros pueden influenciar directamente en el curado de la resina $y$, consecuentemente, en la calidad de los paneles producidos. El pH de la madera puede variar entre 3,0 a 5,5 y, según Kelly citado por Trianoski et al. (2015), maderas con un $\mathrm{pH}$ muy ácido pueden causar pre-curado de la resina a base de urea formaldehído durante la fase de cierre de los platos de la prensa, perjudicando el grado de adhesión entre las partículas $\mathrm{y}$, consecuentemente, reducir los valores de las propiedades mecánicas de los paneles. Al respecto Poblete (2001), indica que la mayoría de las especies utilizadas en la fabricación de tableros de partículas tienen valores de $\mathrm{pH}$ que varían entre 2,0 y 5,0 .

El presente trabajo de investigación evalúa la viabilidad técnica de la madera de bolaina (Guazuma crinita Mart) procedente de plantaciones de cuatro años, en la elaboración de tableros de partículas de tres capas así como analiza la influencia de la parafina en sus propiedades físicas y mecánicas.

\section{Material y Métodos}

Para la elaboración de los tableros se utilizaron 10 árboles de Bolaina "Guazuma crinita Mart" de 4 años de edad, procedentes de una plantación a campo abierto en la zona de Meriva, Km. 28 de la carretera Neshuya - Curimaná, distrito de Curimaná, provincia de Padre Abad departamento de Ucayali - Perú (8³8’21” S, 7457’52” O).

La masa especifica básica de la madera se determinó según lo estipulado en la Norma NTP 251.011 (INACAL, 2014), las propiedades químicas de la madera en base a la norma TAPPI 
T $204 \mathrm{~cm}$-17 para los extractivos totales, la norma TAPPI 252 (2016) para el pH de la madera, la norma TAPPI T 222 om-15 para la determinación de lignina (2015), el método de Kurschner y Hoffer para determinar la celulosa y la metodología de Jayme - Wise para la determinación de holocelulosa.

La resina utilizada fue úrea formaldehido con un 55\% de contenido de sólidos, viscosidad Brookfield de 430 cP y pH de 7,8. Se utilizó cloruro de amonio como catalizador, un retardador a base de Amoniaco $\left(\mathrm{NH}_{3}\right)$ y agentes hidrófobos a base de parafina.

Para la manufactura de los paneles aglomerados en el laboratorio, las trozas de bolaina fueron astilladas en un astillador de cuchillas circulares del tipo Pallmann hasta obtener hojuelas de distintas dimensiones; para luego ser convertidas a partículas de menores dimensiones en un molino de martillos, luego fueron secadas hasta un contenido de humedad promedio de $5 \%$ en base a la Norma Técnica Peruana 251.010 (INACAL, 2014). Las partículas se tamizaron y separaron para obtener partículas para la capa externa (tamiz $1,10 \mathrm{~mm}$ ) e interna respectivamente (tamiz de $1,10 \mathrm{~mm}$ a 2,00 $\mathrm{mm})$. Luego fueron tamizadas usando la malla número 0,5 para separar los finos o polvillos.

Los paneles fueron producidos con una formación en multicapa, con proporciones en las capas externas e interna de 18:64:18, utilizando una resinosidad de $12 \%$ para las capas externas y $6 \%$ para la capa interna; utilizando un $0,5 \%$ de parafina en la formulación de la cola para la producción de los tableros que llevaron este aditivo.

Los cálculos de los materiales fueron definidos para dimensiones de panel de $40 \mathrm{~cm}$ x $40 \mathrm{~cm}$ x $1,2 \mathrm{~cm}$ y una masa especifica nominal de $0,65 \mathrm{~g} \cdot \mathrm{cm}^{-3}$. Los paneles fueron prensados a una temperatura de $165^{\circ} \mathrm{C}$, presión específica de $10,0 \mathrm{~kg} / \mathrm{cm}^{2}$ y un tiempo de prensado de 6 minutos. Se elaboraron 4 tableros por tratamiento, que representaron un total de 8 tableros experimentales.
Luego de la fabricación de los paneles estos fueron conducidos a la cámara de climatización con condiciones ambientales controladas ( $\mathrm{T}: 20 \pm 2^{\circ} \mathrm{C}$ y HR: $65 \pm 5 \%$ ) hasta llegar a la humedad de equilibrio.

En la Tabla 1 se muestra la cantidad de insumos y materiales utilizados en la preparación de las capas externas e interna de los tableros de partículas con y sin parafina.

Tabla 1. Cantidad de insumos y materiales empleados en las capas externas e interna para tableros con parafina y sin parafina.

Table 1. Quantity of inputs and materials used in the outer and inner layer for boards with paraffin and without parafin.

\begin{tabular}{lllll}
\hline \multirow{2}{*}{ Insumos } & \multicolumn{2}{l}{ Con parafina } & \multicolumn{2}{l}{ Sin parafina } \\
& Capa & Capa & Capa & Capa \\
& Externa & Interna & externa & Interna \\
\hline Partículas & $577 \mathrm{~g}$ & $680 \mathrm{~g}$ & $577 \mathrm{~g}$ & $680 \mathrm{~g}$ \\
Resina & $100,3 \mathrm{ml}$ & $58,3 \mathrm{ml}$ & 100,3 & $58,3 \mathrm{ml}$ \\
& & & $\mathrm{ml}$ & \\
Endurecedor & $1,3 \mathrm{ml}$ & $4,6 \mathrm{ml}$ & $1,3 \mathrm{ml}$ & $4,7 \mathrm{ml}$ \\
Retardador & $2,0 \mathrm{ml}$ & $0,8 \mathrm{ml}$ & $2,0 \mathrm{ml}$ & $0,8 \mathrm{ml}$ \\
Agua & $11,9 \mathrm{ml}$ & - & $18,0 \mathrm{ml}$ & $6,1 \mathrm{ml}$ \\
Parafina & $6,3 \mathrm{ml}$ & $7,2 \mathrm{ml}$ & - & - \\
\hline
\end{tabular}

Las propiedades físicas y mecánicas de los paneles fueron determinadas de acuerdo con la metodología propuesta por las Normas EN 310 (EN, 2002), EN 317 (EN, 2002), EN 319 (EN, 2002c) y EN 323 (EN, 2003), respectivamente para flexión estática (FE), Absorción de agua e hinchamiento en espesor (IE) después de 2 y 24 horas, tracción perpendicular (TP) y masa específica aparente. Fueron ensayados para cada tratamiento 16 probetas para masa específica, 20 probetas para flexión estática, 16 probetas para tracción perpendicular y 40 para hinchamiento en espesor después de 2 y 24 horas. Los valores experimentales fueron comparados con los requisitos propuestos por la Norma EN 312 (EN, 2010).

Los datos obtenidos fueron evaluados estadísticamente mediante un diseño completamente al azar (DCA), realizando un análisis de variancia y una prueba $\mathrm{T}$ para para determinar el nivel de variabilidad entre los valores obtenidos en pruebas físicas y mecánicas de cada tratamiento y los valores promedios entre tratamientos, respectivamente. 


\section{Resultados e Discusión}

La Tabla 2 presenta los valores promedios de masa especifica básica de la madera, la masa específica del tablero y la razón de compactación.

Tabla 2. Masa específica de la madera y razón de compactación de los tableros de partículas.

Table 2. Specific mass of wood and compaction ratio of particleboards.

\begin{tabular}{cccc}
\hline Tratamiento & $\begin{array}{c}\text { MEM } \\
\left(\mathrm{g} . \mathrm{cm}^{-3}\right)\end{array}$ & $\begin{array}{c}\text { MEP } \\
\left(\mathrm{g} . \mathrm{cm}^{-3}\right)\end{array}$ & RC \\
\hline SP & & $0,65 \mathrm{a}$ & $1,55 \mathrm{~b}$ \\
& 0,42 & $(0,88)$ & $(0,88)$ \\
CP & $(0,57)$ & $0,64 \mathrm{a}$ & $1,52 \mathrm{~b}$ \\
& & $(0,48)$ & $(0,48)$ \\
\hline
\end{tabular}

SP: sin parafina; CP: con parafina; MEM: masa especifica de la madera; MEP: masa específica del panel; RC: razón de compactación. Medias seguidas de una misma letra en una misma columna son estadísticamente iguales por el Test de Tukey a $95 \%$ de probabilidad. Valores entre paréntesis se refieren al Coeficiente de variación.

La masa específica básica promedio de las muestras de bolaina utilizadas fue de $0,42 \mathrm{~g} . \mathrm{cm}^{-3}$. El valor promedio de la razón de compactación obtenidos en relación a la masa específica del panel y la masa especifica de la madera fue de 1,5 para los tableros elaborados sin parafina y de 1,4 para los elaborados con parafina.

El valor de masa específica básica promedio se encuentra dentro del rango de 0,3 g. $\mathrm{cm}^{-3}$ a $0,5 \mathrm{~g} . \mathrm{cm}^{-3}$ establecido por Maloney (1993). La razón de compactación de 1,55 para los tableros de partículas elaborados sin parafina y 1,54 para los elaborados con parafina, se encuentra dentro del rango establecido por Poblete (2001) y superior al valor mínimo propuesto por Iwakiri (2010), quien además menciona que la masa específica de la madera es un requisito básico en la selección de especies para la producción de paneles aglomerados, por influenciar directamente en su razón de compactación. Los valores de compactación están comprendidos dentro del rango obtenido por Vital et al. mencionado por Trianoski et al (2015), quien obtuvo una razón de compactación de 1,2 a 1,6 para tableros aglomerados producidos con Virola spp., especie de madera tropical Amazónica con masa específica aparente de $0,43 \mathrm{~g} \cdot \mathrm{cm}^{-3}$. En la Tabla 3 se muestran las características químicas de la madera bolaina.

Tabla 3. Valores promedios de la composición química básica de la madera de Bolaina.

Table 3. Average values of the basic chemical composition of Bolaina wood.

\begin{tabular}{cc}
\hline Características químicas & Valores promedios \\
\hline Extractivos totales & $1,80 \%$ \\
Holocelulosa & $79,23 \%$ \\
Celulosa & $51,85 \%$ \\
Hemicelulosa & $27,38 \%$ \\
Lignina & $28,61 \%$ \\
pH & 6,76 \\
\hline
\end{tabular}

El contenido de extractivos totales se encuentra en el rango hallado por Bueno (1990), que puede oscilar entre 1,00\% y 7,54 $\%$; el contenido de celulosa se encuentra cerca del establecido por Egoavil (1995), de 53,48\%, y por la FAO (1980) de 49,90\%. Esto se puede atribuir a la ubicación geográfica, condiciones medioambientales de crecimiento y a la edad del árbol; para la hemicelulosa el valor encontrado es similar al establecido por FAO, de 27,30 \%, pero inferior a los resultados de Egoavil (1995) de 18,87 \%. El contenido de lignina estuvo por encima de lo establecido por FAO (1980) de 16 \% y Egoavil (1995) de $24,48 \%$, pero dentro de los rangos establecidos por Bueno (1990) de 20,36 \% a 36,65 \%. El pH promedio supera al rango indicado por Marra citado por Trianoski et al. (2015) que considera que el $\mathrm{pH}$ de la madera debe estar entre 3,0 a 5,5 y al rango de 2 a 5 establecido por Poblete (2001). El mismo autor menciona que son escasas las especies que poseen un $\mathrm{pH}$ mayor de 7 y que puedan dar problemas en la fabricación del tablero de partículas.

En la Tabla 4 se presentan los valores promedio de las propiedades físicas de los tableros de partículas elaborados sin y con parafina. 
Tabla 4. Valores promedios de las propiedades físicas de los tableros de partículas.

Table 4. Average values of the physical properties of particleboards.

\begin{tabular}{cccc}
\hline Tratamiento & Propiedad & Unidades & $\begin{array}{c}\text { Valor } \\
\text { promedio }\end{array}$ \\
\hline \multirow{5}{*}{ Sin parafina } & ME & g.cm ${ }^{-3}$ & $0,65 \mathrm{a}(0,88)$ \\
& CH & $\%$ & $8,45 \mathrm{~b}(1,18)$ \\
& HE $24 \mathrm{~h}$ & $\%$ & $26,46 \mathrm{c}(6,72)$ \\
& & $\%$ & $36,41 \mathrm{~d}$ \\
Con parafina & $\mathrm{ME}$ & $\mathrm{g.cm}^{-3}$ & $0,64 \mathrm{a}(0,48)$ \\
& $\mathrm{CH}$ & $\%$ & $8,75 \mathrm{~b}(3,55)$ \\
& $\mathrm{HE} 2 \mathrm{~h}$ & $\%$ & $21,17 \mathrm{c}(3,46)$ \\
& $\mathrm{HE} 24 \mathrm{~h}$ & $\%$ & $38,29 \mathrm{~d}$ \\
& & & $(4,77)$ \\
\hline
\end{tabular}

ME: masa específica; $\mathrm{CH}$ : contenido de humedad; $\mathrm{HE}$, hinchamiento en espesor después de 2 y 24 horas. Medias seguidas de una misma letra en una misma columna son estadísticamente iguales por el Test de Tukey a 95\% de probabilidad. Valores entre paréntesis se refieren al Coeficiente de variación.

Respecto a la masa específica para los tableros sin parafina, se tiene un valor promedio de 0,65 g. $\mathrm{cm}^{-3}$, mientras que para los tableros elaborados con parafina el valor fue de 0,64 g.cm 3; dichos valores se encuentran dentro del valor teórico establecido al elaborar este tipo de tablero de masa especifica media $\left(0,65 \mathrm{~g} \cdot \mathrm{cm}^{-3}\right)$. Asimismo, el análisis de varianza y la prueba $\mathrm{T}$ indican que no existen diferencias significativas entre los valores de los tableros elaborados sin parafina y con parafina.

Respecto al contenido de humedad, los valores promedio para los tableros elaborados sin parafina fue de $8,45 \%$ y para los tableros elaborados con parafina fue de $8,75 \%$, valores están por encima de valor teórico establecido de $8 \%$. Asimismo, los resultados del análisis de varianza y la prueba $\mathrm{T}$ indican que no existen diferencias significativas entre los valores de los tableros elaborados sin parafina y los elaborados con parafina.

Los valores promedio del hinchamiento a las 2 horas y 24 horas, para los tableros elaborados sin parafina fueron de 26,46 $\%$ y $36,41 \%$ respectivamente; asimismo, para los tableros elaborados con parafina fueron de $21,17 \%$ y de $38,29 \%$ respectivamente. Estos valores son elevados, sobre todo para los tableros estructurales utilizados en ambiente húmedo; sin embargo, esta propiedad no es considerada por la Norma EN 312:2010 (CEN, 2010) para los tableros de uso en interiores Tipo P2. El análisis de varianza demuestra que no existen diferencias significativas entre valores de los tableros elaborados con y sin parafina.

Los resultados del hinchamiento a las 2 y 24 horas fueron relativamente elevados, y puede deberse a la aplicación de la misma presión específica para partículas de madera madura a partículas de maderas de 4 años de edad lo cual podría haber afectado su estructura anatómica. No obstante, estos valores se encuentran dentro de los rangos determinados por Trianoski et al. (2015). La norma UNE-EN 312 (CEN, 2010) no considera esta propiedad física para tableros de uso en interiores Tipo P2.

La Tabla 5 y las Figuras 1 y 2 muestran los valores promedio de las propiedades mecánicas de los tableros.

Tabla 5. Valores promedio de las propiedades mecánicas de los tableros de partículas.

Table 5. Average values of the mechanical properties of particleboards.

\begin{tabular}{|c|c|c|}
\hline Tratamiento & Propiedad & $\begin{array}{c}\text { Valor promedio } \\
(\mathrm{MPa})\end{array}$ \\
\hline \multirow{4}{*}{ Sin parafina } & MOR & $15,55 \mathrm{a}$ \\
\hline & & $(3,86)$ \\
\hline & $\mathrm{TP}$ & $0.97 b$ \\
\hline & & $(4,90)$ \\
\hline \multirow{4}{*}{ Con parafina } & MOR & $15,05 \mathrm{a}$ \\
\hline & & $(3,49)$ \\
\hline & $\mathrm{TP}$ & $0,82 b$ \\
\hline & & $(6,53)$ \\
\hline
\end{tabular}

MOR: módulo de ruptura; TP: tracción perpendicular à la superfície. Medias seguidas de una misma letra en una misma columna son estadísticamente iguales por el Test de Tukey a 95\% de probabilidad. Valores entre paréntesis se refieren al Coeficiente de variación.

A partir de los resultados presentados en la Tabla 5 y las Figuras 1 y 2 , se observa que el análisis de varianza y la prueba $\mathrm{T}$ indican que no existen diferencias significativas entre los valores de los tableros elaborados sin parafina y los elaborados con parafina. El módulo de rotura en flexión estática para los 
tableros elaborados sin parafina fue de $15,55 \mathrm{MPa}$ y para los tableros elaborados con parafina de 15,05 $\mathrm{MPa}$, valores que superan en $41 \%$ y $37 \%$ respectivamente, al valor mínimo exigido por la Norma EN 312:2010 (EN, 2010) (11,00 MPa) para tableros de uso en interiores Tipo P2.

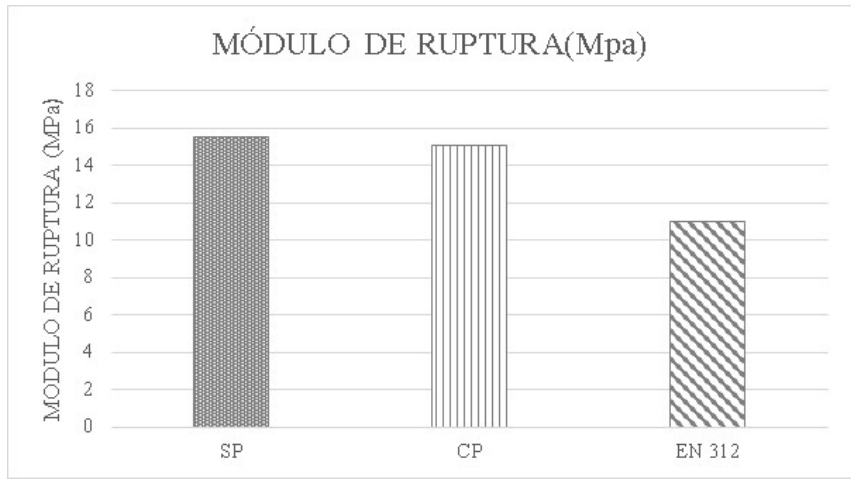

SP:sin parafina; CP:con parafina; EN 312:2010

Figura 1. Resistencia a la flexión estática de los tableros de bolaina.

Figure 1. Static bending of bolaina boards.

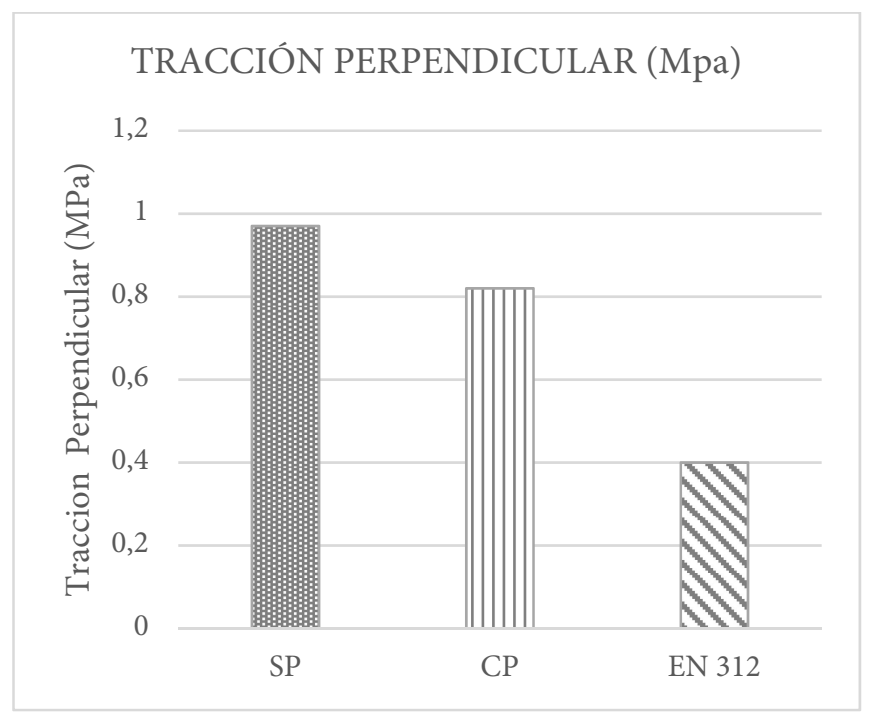

SP: sin parafina; CP: con parafina; EN 312:2010.

Figura 2. Resistencia a la tracción perpendicular a la superficie de los tableros de bolaina.

Figure 2. Internal bond to the surface of bolaina boards.

En general, los valores de MOR en flexión estática obtenidos en la investigación, se encuentran comprendidos dentro del rango determinado por Trianoski et al. (2011) de $10,51 \mathrm{MPa}$ a $18,73 \mathrm{MPa}$, al evaluar especies alternativas de rápido crecimiento para la producción de tableros de partículas de tres capas, Iwakiri et al. (2012) de 11,55 MPa a
20,15 MPa al evaluar el efecto de diferentes porcentajes de resina en la producción de tableros de partículas homogéneos y multicapa de Melia azederach (CINAMOMO) y Pinus taeda., Iwakiri et al. (2010) de 12,03 $\mathrm{MPa}$ a 16,65 $\mathrm{MPa}$, al evaluar el potencial de utilización de la madera de Schizolobium amazonicum "Parica" y Cecropia hololeuca "Embaúba" para la producción de tableros de partículas y Trianoski et al. (2015) de 13,51 MPa a 18,23 $\mathrm{MPa}$, en su investigación sobre la producción de tableros de partículas con cuatro especies de maderas tropicales de la Amazonía.

Así mismo los valores de MOR en flexión estática obtenidos fueron superiores a los determinados por Trianoski et al. (2013) de 12,88 MPa a 13,09 MPa, al evaluar las propiedades físicas y mecánicas de tableros de partículas de Acrocarpus fraxinifolius, elaborados con diferentes porcentajes de corteza e inferiores a los valores obtenidos por Zambrano et al. (2013) de 21.11 MPa a 26.08 MPa al evaluar la resistencia de los tableros de partículas fabricados con residuos industriales de madera de Pinus patula.

En relación a la tracción perpendicular a la superficie cuyos valores se muestran en la Tabla 5, el análisis de varianza y la prueba $\mathrm{T}$ determinaron que no existen diferencias significativas entre los valores de tracción perpendicular de los tableros elaborados sin parafina y con parafina. La tracción perpendicular a la superficie de los tableros elaborados sin parafina fue de 0,97 MPa, mientras que para los elaborados con parafina el resultado fue de 0,82 $\mathrm{MPa}$, valores que fueron superiores al valor mínimo exigido por la norma EN 312:2010 $(\mathrm{EN}, 2010)(0,40 \mathrm{MPa})$ para tableros de uso en interiores Tipo P2.

En general, los valores de adhesión interna obtenidos en ésta investigación están dentro de los intervalos determinados por Trianoski et al (2011) de 0,83 $\mathrm{MPa}$ a 1,20 $\mathrm{MPa}$, en su evaluación de especies alternativas de rápido crecimiento para la producción de tableros de partículas de tres capas e Iwakiri et al. (2012) de 0,43 MPa a 1,55 MPa, al evaluar el efecto de diferentes porcentajes de resina en la producción de tableros 
de partículas homogéneos y multicapa de Melia azederach (CINAMOMO) y Pinus taeda.

Así mismo los valores de adhesión interna obtenidos fueron superiores a los determinados por Iwakiri et al. (2010) de 0,58 $\mathrm{MPa}$ a 0,88 $\mathrm{MPa}$, al evaluar el potencial de utilización de la madera de Schizolobium amazonicum "Parica" y Cecropia hololeuca "Embaúba” para la producción de tableros de partículas, Trianoski et al. (2015) de 0,54 MPa a 0,58 MPa, en su investigación sobre la producción de tableros de partículas con cuatro especies de maderas tropicales de la Amazonia y Zambrano et al. (2013) de 0,37 MPa a 0,45 MPa al evaluar la resistencia de los tableros de partículas fabricados con residuos industriales de madera de Pinus patula.

\section{Conclusiones}

Los paneles aglomerados producidos con madera de Guazuma crinita Mart. (Bolaina) con la adición de parafina y sin parafina presentaron propiedades mecánicas superiores a los requisitos mínimos establecidos en la norma EN 312:2010.

Los valores de hinchamiento a las 2 y 24 horas fueron superiores a los valores exigidos por norma EN 312:2010 para tableros no estructurales utilizados en ambiente húmedo (P3).

El parafinado no influyó significativamente en las propiedades físicas y mecánicas de los tableros aglomerados, por lo que, por costos de fabricación, se podría prescindir de este aditivo con esta especie de madera.

Los resultados de esta investigación indican que la madera de Guazuma crinita Mart (Bolaina) es técnicamente viable para la producción de paneles aglomerados.

\section{Referencias}

BUENO, J. Tipos de Pulpa para Papel. II Symposium de la Industria de Pulpa y Papel. UNALM. Asociación Peruana de Pulpa y Papel, Lima, 1990, 238 p.
CEN - EUROPEAN COMMITTEE FOR STANDARDIZATION. EN 312. Particleboards Specifications. CEN, Brussels, 2010.

CEN - EUROPEAN COMMITTEE FOR STANDARDIZATION. EN 310. Determination of modulus of elasticity in bending and of bending strength. CEN, Portugal, 2002.

CEN - EUROPEAN COMMITTEE FOR STANDARDIZATION. EN 317. Determination of swelling in thickness after immersion in water. CEN, Brussels, 2002.

CEN - EUROPEAN COMMITTEE FOR STANDARDIZATION. EN 319. Determination of tensile strength perpendicular to the faces of the plate. CEN, Lisboa, 2002c.

CEN - EUROPEAN COMMITTEE FOR STANDARDIZATION. EN 323. Determination of board density. CEN, Brussels, 2003.

EGOAVIL, C. G. Aptitud de Pulpa Química al Sulfato de Bolaina Blanca ( Guazuma crinita) a la fabricación de Papel de Escritura. Revista Forestal del Perú. Vol. XXII, n. 2, 1995.

FAO. Pulping and Paper Makings Properties of Fast Growing Plantation Wood Species. Roma, Italia, 1980.

FLORES, B. Y. Árboles nativos de la Región Ucayali. Instituto Nacional de Innovación Agraria (INIA). Estación Experimental Agraria Pucallpa, Perú, p. 375. 2018.

INSTITUTO NACIONAL DE LA CALIDAD. NTP 251.010. Madera. Método para determinar el contenido de humedad. Lima, 2014.

INSTITUTO NACIONAL DE LA CALIDAD. NTP 251.011. Madera. Método para determinar la densidad. Lima, 2014.

IWAKIRI, S.; MONTEIRO, J. L.; TRIANOSKI, R.; PRATA, J. G. Production of homogeneus and multilayer particleboard from Melia azederach (CINAMOMO) and Pinus taeda with different resin contents. Cerne, Lavras, v. 18, n. 3, p. 465-470. 2012.

IWAKIRI, S.; VIANEZ, B. F.; SAKS, WEBER, C.; TRIANOSKI, R.; ALMEIDA, V. C. Evaluation of potential use of the wood of Schizolobium amazonicum "Parica" and Cecropia hololeuca "Embaúba" to particleboard manufacture. Acta Amazónica vol. 40, n. 2, p. 303-308, 2010.

MALONEY, M. T. Modern particleboard and dry process fiberboard manufacturing. San Francisco; Miller Freeman, 689 p, 1993. 
MOSLEMI, A. A. Particleboard. Vol I, Southern Illinois University Press, 245 p. 1974.

POBLETE, H. Tableros de Partículas. Valdivia. Chile. 177 p, 2001.

PUTZEL, L.; CRONKLETON, P.; LARSON, A.; PINEDOVÁSQUEZ, M.; SALAZAR, O. y SEARS, R. Producción y comercialización de bolaina (Guazuma crinita), una especie amazónica de rápido crecimiento. Boletín CIFOR. No 25,6 p, 2013.

RAMOS-HUAPAYA, A. E. y DOMINGUEZ, G. Selección de árboles de Bolaina blanca (Guazuma crinita Mart.) como candidatos a árboles "Plus" para ensayos de rejuvenecimiento y brotación. Ecología Aplicada. v. 15 n. 2. Lima. 10 p, 2016.

SERVICIO NACIONAL FORESTAL Y DE FAUNASILVESTRE-SERFOR. Anuario Forestal y de fauna silvestre 2016. Lima: Ministerio de Agricultura y Riego, 107 p,2018.

SERVICIO NACIONAL FORESTAL Y DE FAUNASILVESTRE-SERFOR. Anuario Forestal y de fauna silvestre 2017. Lima: Ministerio de Agricultura y Riego, 124 p, 2019.

TECHNICAL ASSOCIATION OF THE PULP AND PAPER INDUSTRIA, TAPPI T 252 om-16: $\mathrm{pH}$ an electrical conductivity of water extracts of pulp, paper, and paperboard. Atlanta, 2016.

TECHNICAL ASSOCIATION OF THE PULP AND PAPER INDUSTRIA, TAPPI T $204 \mathrm{~cm}-17$ : Solvent extractives of Wood and Pulp. Atlanta, 2017.

TECHNICAL ASSOCIATION OF THE PULP AND PAPER INDUSTRIA, TAPPI T 222 om-17: Acid-insoluble lignin in wood and pulp. Atlanta, 2017.

TRIANOSKI, R.; IWAKIRI, S.; D NASCIMENTO, C. C.; BILA, N. F. Particleboard manufacture from four species of tropical timber of the Amazon region. Scientia Forestalis, v. 43, n. 106. p. 445-452, 2015.

TRIANOSKI, R.; IWAKIRI, S.; MONTEIRO J. L.; PRATA, J. G. Physical and mechanical properties of particleboars of Acrocarpus fraxinifolius compounds with different percentages of bark. Ciência Florestal, Santa Maria v. 23, n. 4, p. 761-769, 2013.

TRIANOSKI, R.; IWAKIRI, S.; MONTEIRO J. L..; PRATA, J. G. Evaluation of fast growing alternatives species to threelayer particleboard manufacture. Scientia Forestalis, Piracicaba, v. 39, n. 89, p. 097-104, 2011.
WIGHTMAN, K. E; CORNELIUS, J. P.; UGARTEGUERRA, L.J. AG. ¡Plantemos madera! Manual sobre el establecimiento, manejo y aprovechamiento de plantaciones maderables para productores de la Amazonía peruana. World Agroforestry Centre (ICRAF). Technical Manual n. 4. p. 193, 2006.

ZAMBRANO, L.; MORENO, P.; MUÑOZ F.; DURAN, J.; GARAY, D.; VALERO S. Particleboard manufacture from industrial residues of Pinus patula wood. Madera y Bosques, v. 19, n. 3 , p. $65-80,2013$. 